\title{
Acute and preventive pharmacological treatment of post-traumatic headache: a systematic review
}

\author{
Eigil Lindekilde Larsen ${ }^{1 \dagger}$, Håkan Ashina ${ }^{1 \dagger}$, Afrim Iljazi ${ }^{1}$, Haidar Muhsen Al-Khazali', Kristoffer Seem', \\ Messoud Ashina ${ }^{1}$, Sait Ashina ${ }^{2+}$ and Henrik Winther Schytz ${ }^{1 *+}$ (D)
}

\begin{abstract}
Background: Post-traumatic headache (PTH) is associated with considerable disability and reduced health-related quality of life. Despite the very high prevalence of PTH, there are no evidence-based guidelines for PTH treatment. Thus, we found it timely to provide a systematic review of the current literature on acute and preventive pharmacological treatment of PTH using PubMed and Embase databases.

Findings: Included studies involved acute and preventive pharmacological treatment of headache attributed to traumatic injury to the head in adherence to the International Classification of Headache Disorders (ICHD) criteria. Of 1424 potentially relevant articles identified, 63 were retrieved for detailed evaluation and seven studies (one prospective and six retrospective) met the inclusion criteria. None of the seven included studies were randomized clinical trials (RCTs) or used a placebo-controlled study design.

Conclusion: We found that there is a lack of high-quality evidence-based studies on the pharmacological treatment of PTH. Future studies are highly needed and must emphasize open-label studies with rigorous methodology or RCTs with a placebo-controlled design.
\end{abstract}

Keywords: Head injury, Post-traumatic headache, Treatment, Traumatic brain injury, Concussion, Systematic review

\section{Introduction}

Post-traumatic headache (PTH) is a common secondary headache disorder [1] associated with considerable disability and reduced health-related quality of life [2]. Epidemiological data has shown a lifetime PTH prevalence of $4.7 \%$ in men and $2.4 \%$ in women [3], with migraine-like and tension-type-like headache being the most common headache phenotypes [4]. In addition, a substantial number of PTH patients experience disabling comorbidities such as symptoms of depression, anxiety, and sleep disturbances [5].

In the International Classification of Headache Disorders (ICHD) [6], PTH is defined by onset of headache

\footnotetext{
* Correspondence: henrik.winther.schytz.01@regionh.dk

${ }^{\dagger}$ Eigil Lindekilde Larsen and Håkan Ashina are co-first authors.

'Sait Ashina and Henrik Winther Schytz are co-senior authors.

${ }^{1}$ Danish Headache Center, Department of Neurology, Rigshospitalet Glostrup,

Faculty of Health and Medical Sciences, University of Copenhagen,

Copenhagen, Denmark

Full list of author information is available at the end of the article
}

within seven days following trauma or injury to the head and/or neck and is also further characterized as either acute (the first three months from headache onset) or persistent - the latter being if the headache persists beyond 3 months.

Despite a very high prevalence of $\mathrm{PTH}$, there are no evidence-based guidelines for acute or preventive pharmacological treatment of PTH. The likelihood of patients receiving optimal treatment is therefore low, with a high risk of unnecessary treatment exposure. $\mathrm{Pa}$ tients with very frequent or daily headache following a trauma are also at risk of developing medication-overuse headache $(\mathrm{MOH})[3]$.

Current pharmacological treatments for PTH are based on acute or preventive medications used for primary headache disorders $[4,5]$, since PTH often mimics a migrainelike or tension-type headache-like phenotype [6]. This approach lacks evidence and often results in poor treatment responses [7]. Furthermore, side effects to pharmacological 
treatment may conflict with PTH comorbidities such as depression and anxiety. Here, we review the current literature on acute and preventive treatment of PTH and provide a useful overview for clinicians. In addition, we address methodological limitations and provide recommendations for future research.

\section{Methods}

\section{Data sources}

We searched PubMed and Embase databases for articles on acute and prophylactic pharmacological PTH treatment. The search was performed on January 16, 2019 with the following search string: (post traumatic headache OR post-traumatic headache OR posttraumatic headache OR post traumatic migraine OR posttraumatic migraine $O R$ posttraumatic migraine $O R$ post concussion headache OR post-concussion headache OR post concussion migraine OR post-concussion migraine) AND (treatment OR therapy). The search was performed from database inception until the date of the database search. The electronic database search was supplemented with manual searches for published, unpublished and ongoing randomized clinical trials (RCTs) in ClinicalTrials.gov. The search term was "post-traumatic headache".

\section{Selection criteria}

The search was limited to articles on human subjects published in English. We also reviewed the reference lists of relevant primary articles and reviews to identify studies that were missed in the search process. All eligible studies were screened in accordance with the Preferred Reporting Items for Systematic Reviews and Meta-Analyses (PRISMA) reporting guidelines.

\section{Study inclusion and data extraction}

Inclusion and exclusion criteria are presented in Fig. 1. We only included studies in which the subjects fulfilled the diagnostic criteria for headache attributed to traumatic injury to the head in accordance with any version of the International Classification of Headache Disorders (ICHD) [8]. One investigator (E.L.L) screened all articles by title and abstract. Following this, two investigators (E.L.L and A.I.) performed a full-text screening and determined which articles should be included. Another investigator (H.M.A) subsequently reassessed all the included articles. Final inclusion was decided by consensus between the three investigators (E.L.L, A. I and H.M.A). If consensus was not reached, one independent investigator (H.A) was available to provide advice. For each study, two investigators (E.L.L and A.I) recorded data on study design, assessment methods, inclusion

Inclusion Criteria:

- English language articles published in peer-reviewed journals or trials registered in Clinicaltrials.gov

- Male or female subjects with post-traumatic headache defined as headache attributed to traumatic injury to the head in accordance with any version of the International Classification of Headache Disorders

- Clinical studies (randomized, non-randomized or case series), either placebo-controlled or openlabel, evaluating efficacy of pharmacological interventions for acute or persistent post-traumatic headache

- Eligible outcome measures include:

- Change in headache intensity measured by the visual analogue scale (VAS), numerical rating scale (NRS), categorical pain scale or responder rate

- Change in headache frequency reported as: number of days with headache, number of episodes of headache, number of days with moderate to severe headache, number of episodes with moderate to severe headache or responder rate

- Change in duration of headache or duration of headache exacerbations measured as hours or days of continuous pain without relief

- Patient reported outcomes using scales such as the Patient Global Impression of Change scale (PGIC), the Functional Impairment Scale (FIS) or other validated scales

\section{Exclusion Criteria:}

- Abstracts, conference proceedings, reviews, case-reports or meta-analyses

- Animal studies

- Study inclusion dependent on condition, symptom or disorder other than acute or persistent posttraumatic headache

- Eligible outcome measures could not be extracted properly

Fig. 1 Eligibility criteria 
criteria, exclusion criteria, age, gender, total number of subjects, headache phenotype, outcome measures and other data relevant for the scope of this review.

\section{Results}

The database search resulted in a total of 1218 search hits (Fig. 2). Three additional articles were identified through a manual search of the reference lists of relevant primary articles and reviews. A total of 1221 articles were screened by title and abstract of which 63 articles were retrieved and assessed for eligibility in the present study. Seven articles met the inclusion criteria and were included in the qualitative synthesis [9-15]. Four studies [9-12] reported on acute PTH treatment, two studies $[13,14]$ on preventive PTH treatment and one study [15] on both acute and preventive PTH treatment. The studies were very heterogeneous in terms of the study populations assessed. Thus, three of the seven studies consisted of pediatric populations $[9,10,14]$, one study was in children or adolescents [12] and one was in a population with both adults and children [13]. The rest consisted of a study on military personnel [15], and one study in an adult population [11]. None of the seven included studies were RCTs or included a placebocontrolled study design. Six studies collected data retrospectively $[9,10,12-15]$ while one study acquired data prospectively [11].

Here, we present data focusing first on acute pharmacological treatment of PTH and then on preventive pharmacological treatment of PTH. Table 1 presents study characteristics for each of the seven included studies.

\section{Acute pharmacological treatment of post-traumatic headache}

Five studies $[9-12,15]$ investigated acute pharmacological PTH treatment. The list of acute medications included common analgesics such as ketorolac [12], unspecified non-steroidal anti-inflammatory drugs (NSAIDs) [15], acetaminophen [15] and combination drugs (Excedrin, Cafergot and Midrin) [15]. Specific analgesics used were lidocaine [9, 10], triptans [15] and opioids [15]. Antiemetics used were ondansetron [12], metoclopramide [11, 12] and prochlorperazine [12]. Other drugs used were diphenhydramine [11], methylprednisolone acetate [9] and triamcinolone [9].

A retrospective cross-sectional emergency department (ED) based study investigated the treatment response of intravenous acute migraine therapies in an adolescent (aged 8-21 years) group consisting of 254 subjects [12]. All participants presented to the ED within 14 days following mild traumatic brain injury (mTBI). The subjects received one of the following four intravenous (IV) acute treatment options: 1$)$ ketorolac only $(n=55), 2)$ ketorolac and metoclopramide/prochlorperazine $(n=132), 3)$ metoclopramide or prochlorperazine $(n=30)$ and 4$)$ ondansetron only $(n=37)$. The dosages used were not reported. In addition, $95 \%$ of the subjects were discharged from the ED and 37\% received pre-treatment with oral analgesics (acetaminophen or ibuprofen) in the

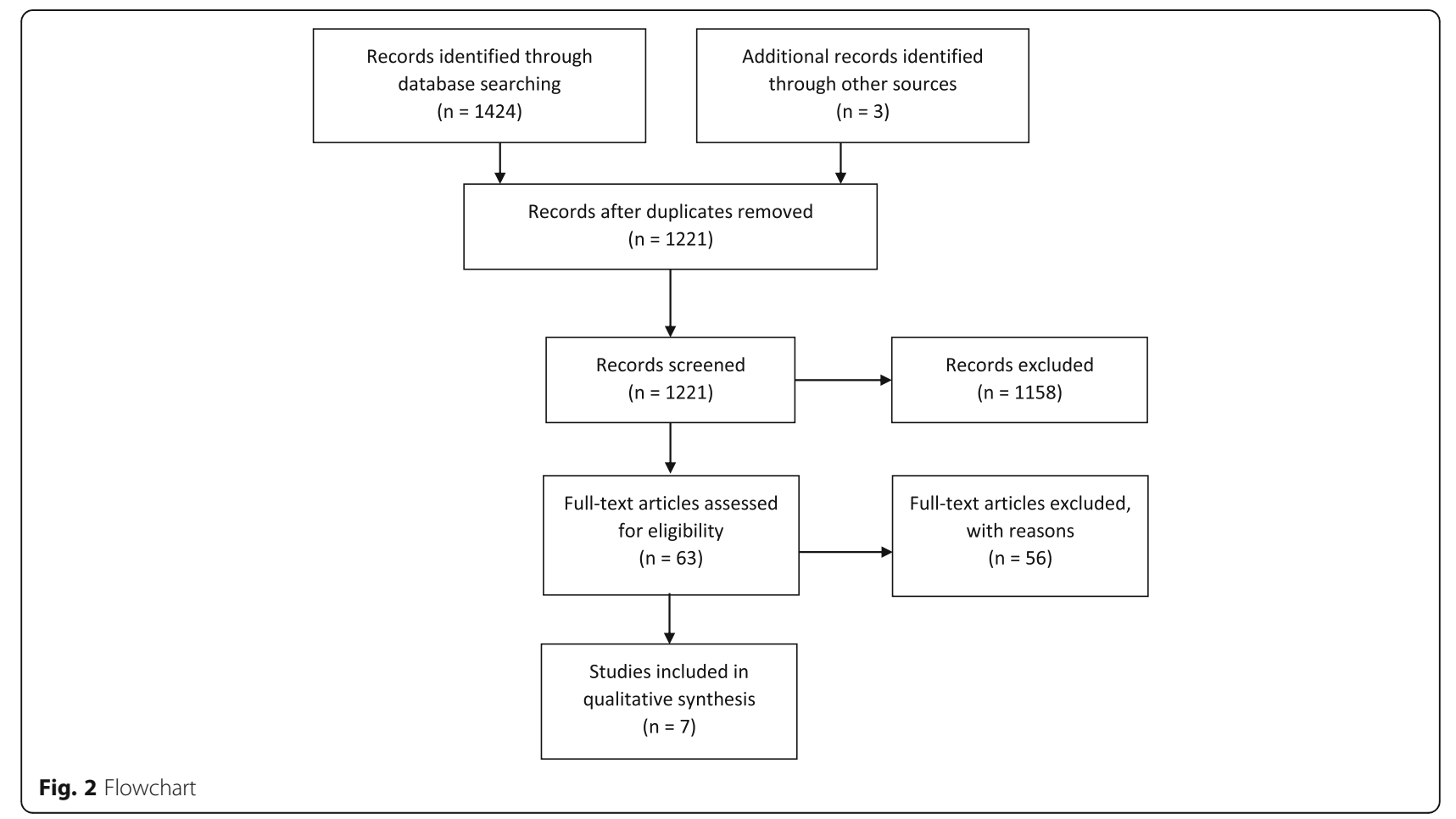



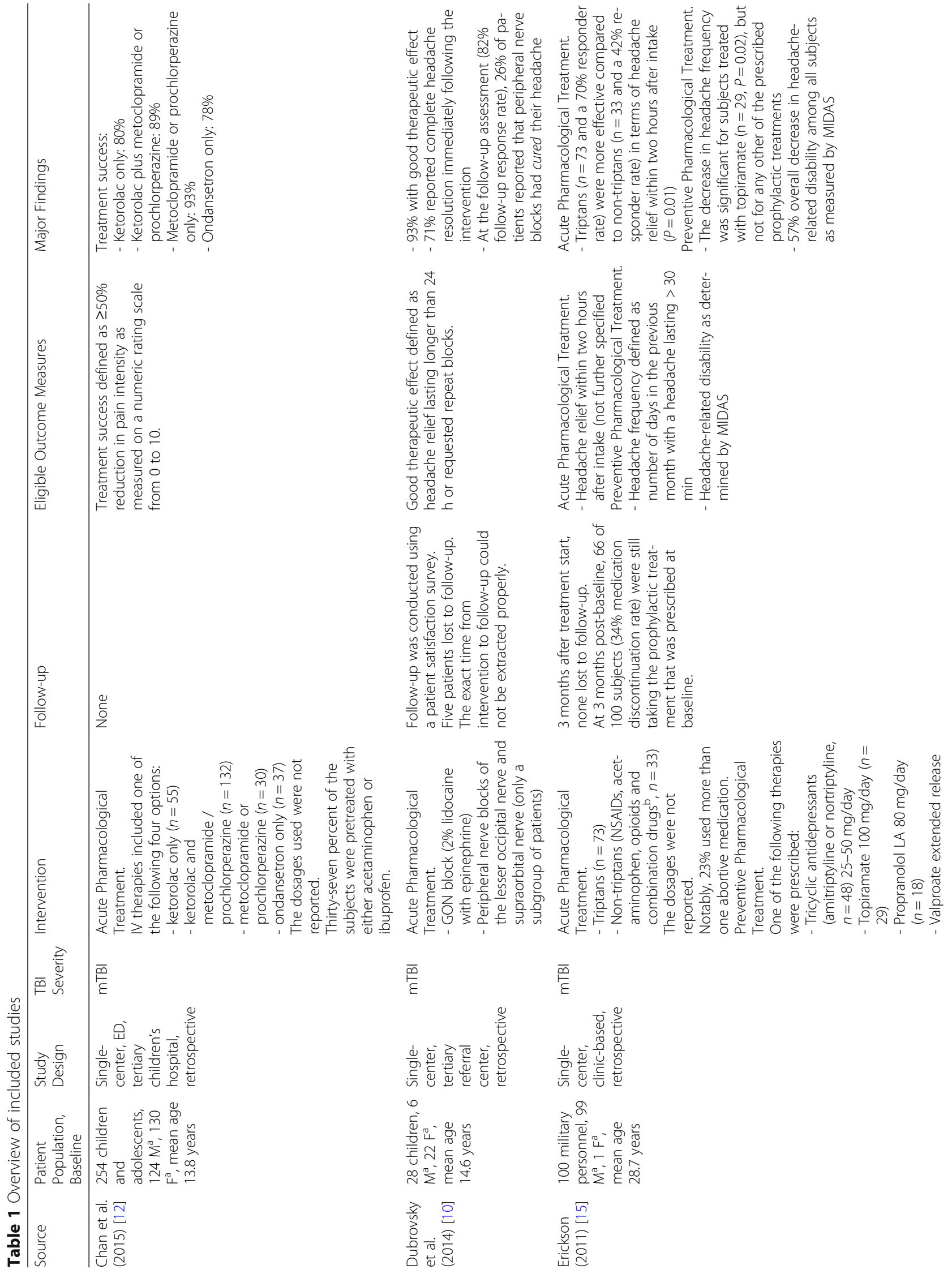

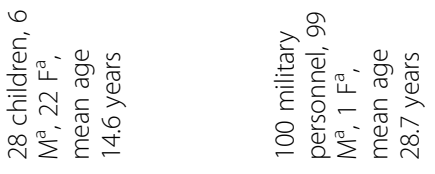

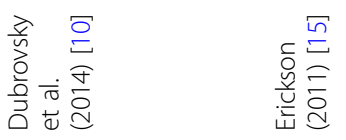




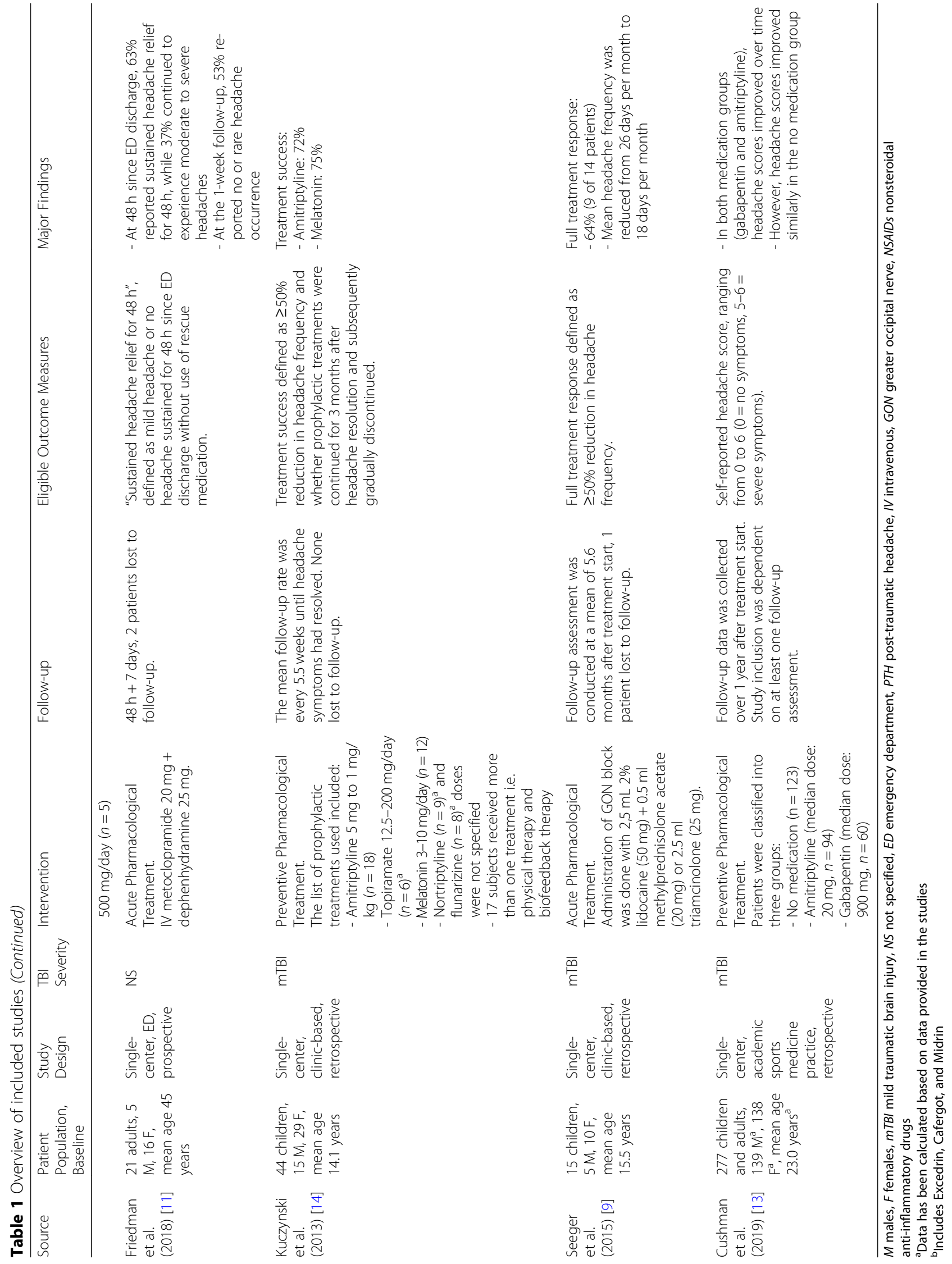


ED. The primary outcome was $\geq 50 \%$ reduction in headache intensity score during the ED visit as measured on a numeric rating scale (NRS) from 0 to 10 . The overall treatment response was $87 \%$ with approximately half of the subjects reporting complete resolution of headache. The treatment response of each of the four drug combinations is described in Table 1.

In a retrospective case series study, 28 children with PTH (aged 10-17 years) reported tenderness over the greater occipital nerve (GON) following palpation [10]. The patients were then treated with peripheral nerve blocks of the scalp, including GON blocks, lesser occipital nerve blocks and supraorbital nerve blocks. The blocks contained 2\% lidocaine with epinephrine. The primary outcome was to examine the proportion of patients with good effect (pain relief $>24 \mathrm{~h}$ and/or repeat blocks requested), partial effect (pain relief $<24 \mathrm{~h}$ and no subsequent request for repeat blocks) or no effect (poor or no pain relief following intervention). Pain relief was defined as $<3 / 10$ pain intensity on a NRS from 0 to 10 . The first peripheral nerve blocks were conducted at a mean of 70 days since the mTBI. Fifty-seven percent of the subjects had a history of multiple concussions, while $70 \%$ had a history of headache prior to the mTBI. Ninety-three percent of the subjects reported good therapeutic effect, while the rest reported partial effect. In addition, $71 \%$ of the subjects reported complete headache resolution following the intervention. A patient satisfaction survey was conducted post-intervention (82\% follow-up response rate) with $26 \%$ of subjects reporting that peripheral nerve blocks had cured their headache. The time interval from treatment start to survey response was not further specified. Moreover, the authors reported that $82 \%$ received intravenous metoclopramide treatment prior to the first treatment with peripheral nerve blocks. Following metoclopramide treatment, 32\% of subjects reported complete immediate headache resolution while $44 \%$ achieved partial and $24 \%$ no headache relief, respectively. Intravenous metoclopramide treatment was conducted at a mean of 39 days since the mTBI. The authors did not report the dosages used for intravenous metoclopramide treatment.

In another case series, Seeger et al. [9] performed a retrospective chart review in 15 children (mean age: 15; range 13-17) with $\mathrm{PTH}$ attributed to $\mathrm{mTBI}$, who were treated with GON blocks. Follow-up data was obtained from 14 of 15 subjects at 6 months after the intervention. The time range from injury to intervention was 112 months with 11 subjects reporting persistent PTH (duration $>3$ months). The primary outcome was "full response" defined as $\geq 50 \%$ reduction in headache frequency or "partial response" defined as reduction in headache frequency less than $50 \%$. Sixty-four percent of the subjects reported "full response" at the follow-up assessment, while one subject reported "partial response". Mean headache frequency was reduced from $26 \pm 7$ days per month pre-mTBI to $18 \pm 12$ days per month post-mTBI $(P=0.014)$.

A prospective ED-based open-label study investigated headache relief in 21 patients treated with intravenous metoclopramide $20 \mathrm{mg}$ and diphenhydramine $25 \mathrm{mg}$ after acute PTH attributed to TBI [11]. All patients reported TBI within the past 10 days at the time of intervention, and $90 \%$ provided data at both the $48 \mathrm{~h}$ and one-week follow-up. The primary outcome was "sustained headache relief for $48 h$ ", defined as mild headache or no sustained headache for $48 \mathrm{~h}$ since ED discharge without use of rescue medication. At $48 \mathrm{~h}$ since ED discharge, $63 \%$ reported sustained headache relief for $48 \mathrm{~h}$, while $37 \%$ continued to experience moderate to severe headaches. At the one-week follow-up, 53\% reported no or rare headache occurrence.

An observational retrospective cohort single-center study at a US Army neurology clinic investigated treatment outcomes in 100 military personnel with de novo PTH attributed to mTBI [15]. Seventy-seven percent of the subjects had sustained a blast-related mTBI, while $23 \%$ had suffered a non-blast mTBI. In addition, 52\% screened positive for post-traumatic stress disorder, while $38 \%$ screened positive for depression. More than $90 \%$ of the subjects presented with a migraine-like headache. The mean time since PTH-onset was 18 months, and the mean headache frequency was 17 days per month. This study mainly focused on preventive PTH treatment but did also provide data on acute PTH treatment. The author reported that triptans $(n=73$ and a $70 \%$ responder rate) were more effective compared to non-triptans ( $n=33$ and a $42 \%$ responder rate) in terms of headache relief (not further specified) within two hours after intake $(P=0.01)$. Notably, 23\% used more than one abortive medication.

\section{Preventive pharmacological treatment of post-traumatic headache}

Three studies investigated preventive pharmacological PTH treatment. One study included military personnel [15], one study included a pediatric sample [14] and one study included a combined adult and pediatric population [13]. The list of preventive medications used included: amitriptyline [13-15], nortriptyline $[14,15]$, topiramate $[14,15]$, propranolol [15], valproate [15], melatonin [14], gabapentin [13] and flunarizine [14].

In the aforementioned military study [15], 100 subjects received the following prophylactic medications: tricyclic antidepressants (amitriptyline or nortriptyline, $n=48$ ) $25-50 \mathrm{mg} /$ day, topiramate $(n=29) 100 \mathrm{mg} /$ day, propranolol $(n=18) 80 \mathrm{mg} /$ day and valproate $(n=5) 500 \mathrm{mg} /$ day). Some patients also received non-pharmacological 
treatment (e.g. physical therapy, neuropsychology) during the observational period. Subjects were allowed to use abortive medication such as NSAIDs $(\mathrm{n}=18)$ and triptans $(n=73)$. Primary outcome measures were assessed by phone three months after baseline evaluation and included: 1) number of days in the previous month with a headache lasting more than $30 \mathrm{~min}, 2$ ) headacherelated disability as determined by Migraine Disability Assessment (MIDAS) questionnaire, and 3) whether headaches were reliably relieved within $2 \mathrm{~h}$ after intake of abortive medication. This study found that headache frequency decreases with 2.6 days $(-15.5 \%, P=0.009)$ at follow-up compared to baseline after the subjects started on prophylactic treatment. Thirty-five percent experienced $a \geq 50 \%$ decrease in headache frequency. The decrease in headache frequency was significant for subjects treated with topiramate $(n=29, P=0.02)$, but not for any of the other prescribed prophylactic treatments. At three months post-baseline, 66 of 100 subjects (34\% medication discontinuation rate) were still taking the prophylactic treatment that was prescribed at baseline. Moreover, the study found a 57\% decrease in headacherelated disability among all subjects as measured by MIDAS. In conclusion, the authors stated that topiramate could be an effective prophylactic drug for PTH, while triptans appeared to be the most efficient abortive medication for PTH. However, these findings would need to be validated in prospective, placebo-controlled RCTs.

Kuczynski et al. [14] investigated the efficacy of prophylactic PTH treatment using a retrospective chart review in 44 children (mean age: 14), who attended a brain injury clinic due to PTH. The mean time since traumatic brain injury (TBI) was 7 months (range: 1-29 months) and $61 \%$ reported daily headaches. The list of prophylactic treatments used in the study included: amitriptyline $(5 \mathrm{mg} /$ day to $1 \mathrm{mg} / \mathrm{kg} /$ day), nortriptyline (dosage not specified), topiramate (12.5 to $200 \mathrm{mg} /$ day), melatonin (3-10 mg/day) and flunarizine (dosage not specified). The primary outcome was $\mathrm{a} \geq 50 \%$ reduction in headache frequency and whether prophylactic treatments were continued for 3 months after headache resolution and subsequently gradually discontinued. The authors reported that the overall treatment response was 64\%. More specifically, melatonin and amitriptyline yielded a treatment success rate of $75 \%$ ( 9 of 12 subjects) and $72 \%$ (13 of 18 subjects), respectively. However, 39\% received more than one treatment i.e. physical therapy and biofeedback therapy.

A retrospective cohort study in an academic sports medicine practice classified patients into three groups: gabapentin group (median dose: $900 \mathrm{mg}, n=60$ ), amitriptyline group (median dose: $20 \mathrm{mg}, n=94$ ) and no medication group $(n=123)$ [13]. The latter was defined as subjects who had not been prescribed any pharmacological treatment during the study period. No patient was prescribed both gabapentin and amitriptyline. A 7point numeric rating scale from 0 to 6 ( 0 no headache, 5-6 severe headache) was used to record headache scores. In the gabapentin and amitriptyline groups, most subjects $(\sim 80 \%)$ were prescribed pharmacological treatment at the first or second visits (median time since mTBI: 4.3 weeks) and inclusion was based on at least one follow-up visit after treatment initiation. Therefore, 146 subjects were excluded because they did not have follow-up measurements. The reasons for loss to followup were not available. For the $277 \mathrm{PTH}$ patients that were included in the final analysis, the mean number of physician visits per patient was 4.1 during a mean period of 4.8 months. In both medication groups (gabapentin and amitriptyline), headache scores improved over time. However, headache scores improved similarly in the no medication group.

\section{Discussion}

In conclusion, we found a lack of quality studies on both acute and preventive pharmacological treatment of PTH. Based on data reviewed from seven studies, efficacy of any pharmacological intervention could not be inferred as discussed below. The included studies used an openlabel design limited by both investigator-subject interaction and placebo response. The latter varies widely across clinical trials with both acute and preventive medications for migraine [16-18]. A similar placebo effect could be expected in PTH patients. The primary and secondary endpoints varied between the included studies, making treatment comparisons unfeasible. In addition, 5 of 7 included studies included pediatric populations. In this context, it is worth noting that high placebo responses to pharmacological treatment have previously been reported in pediatric migraine patients [19]. Furthermore, the studies were also considerably underpowered with sample sizes ranging from 15 to 277 patients. In addition, the only prospective study included did not even establish baseline headache characteristics using a prospectively collected headache diary [11]. Some of the reviewed studies included subjects with substantial within-study variance in terms of time range from TBI to intervention [9, 10, 13-15]. As an example, the preventive treatment study [14] included 44 children with a mean time period since TBI of 7 months (range: 1-29 months). Thus, it is highly likely that some of the subjects experienced spontaneous reduction in headache frequency during the treatment period.

\section{Recommendations for future research}

Further research in acute and preventive treatment for PTH is highly warranted. Data analysis can be greatly 
enhanced, if consensus guidelines for controlled trials of acute and preventive treatment of PTH are provided. We suggest several aspects that should be considered to improve data consistency and study design reliability. First, future RCTs should include a double-blinded, randomized, parallel-group study design. This would enable us to prove drug efficacy and provide robust information on safety and tolerability. Second, future open-label studies and RCTs should require included subjects to fulfill the ICHD criteria for PTH to reduce population heterogeneity. A future version of the ICHD may benefit from subdividing PTH based on headache phenotype, as there could be differences in treatment outcome depending on the headache phenotype. Along this line of reasoning, detailed characterization of initial injury severity and type (i.e. GCS or computer tomography/magnetic resonance imaging classification) would also offer much needed patient stratification, which in turn could provide more targeted treatment approaches for individual patients. Third, baseline data on headache characteristics (including headache phenotype), headache 'triggers' (i.e. physical and cognitive activity), comorbid psychiatric illness (e.g. anxiety and depression), number of TBIs, pretrauma history of headache and other relevant data should be recorded prior to intervention. Fourth, primary and secondary endpoints should be similar among future studies. In this context, the International Headache Society (IHS) has provided guidelines for controlled trials of acute and preventive treatment of migraine [20, 21]. These guidelines could serve as inspiration, which would enable us to compare efficacy of acute and preventive migraine drugs between PTH and migraine patients. Lastly, PTH patients often present with a plethora of symptoms (i.e. fatigue, depression, sleep disturbances) and not only headache $[2,22]$. It would be interesting if future RCTs and high-quality open-label studies included secondary endpoints set to investigate drug efficacy on factors such as health-related quality of life, work productivity and levels of anxiety and depression.

\section{Conclusion}

This systematic review has shown a low level of evidence to support any pharmacological treatment of PTH. High-quality RCTs and open-label studies are needed to provide robust evidence of clinical utility. Future research efforts should be driven by rigorous methodology and improved outcome assessment of analytical validity. In addition, PTH patients display a myriad of comorbid symptoms (i.e. depression, anxiety, sleep disturbances). Thus, future studies should include multidimensional outcome scales that cover multiple symptom domains. This will facilitate more targeted treatment approaches that accounts for clinical variability between patients and better reflects the concept of precision medicine.

\begin{abstract}
Abbreviations
ED: Emergency department; GON: Greater occipital nerve; ICHD: International Classification of Headache Disorders; MIDAS: Migraine Disability Assessment; $\mathrm{MOH}$ : Medication-overuse headache; mTBI: Mild traumatic brain injury; NRS: Numeric rating scale; NSAIDs: Nonsteroidal anti-inflammatory drugs; PTH: Post-traumatic headache; RCTs: Randomized clinical trials; TBI: Traumatic brain injury
\end{abstract}

\section{Acknowledgments}

Not applicable.

\section{Authors' contributions}

All authors read and approved the final manuscript.

\section{Funding}

None.

\section{Availability of data and materials}

The datasets used in the present review are available from the corresponding author on reasonable request.

Ethics approval and consent to participate

Not Applicable.

Consent for publication

Not Applicable.

\section{Competing interests}

HWS received speaking fees from Novartis and Teva. SA has received honoraria from Allergan and Teva and consultant fees from Amgen and Allergan. SA received honoraria for lecturing from Allergan and Avanir Pharmaceuticals. MA has received personal fees from Alder

BioPharmaceuticals, Allergan, Amgen, Eli Lilly, Novartis, and Teva. MA also participated in clinical trials as the principal investigator for Alder, Amgen, electroCore, Novartis, and Teva. MA also serves as an associate editor of Cephalalgia, associate editor of Headache and co-editor of the Journal of Headache and Pain. MA is President-elect of the International Headache Society, and General Secretary of the European Headache Federation. MA reports research grants from Lundbeck Foundation, Research Foundation of the Capital Region of Copenhagen, and Novo Nordisk Foundation. The other authors declare no conflicts of interest.

\section{Author details}

${ }^{1}$ Danish Headache Center, Department of Neurology, Rigshospitalet Glostrup, Faculty of Health and Medical Sciences, University of Copenhagen, Copenhagen, Denmark. ${ }^{2}$ Departments of Neurology and Anesthesia, Critical Care, and Pain Medicine, Beth Israel Deaconess Medical Center, Harvard Medical School, Boston, USA.

Received: 18 July 2019 Accepted: 2 October 2019 Published online: 21 October 2019

\section{References}

1. Nampiaparampil DE (2014) Prevalence of chronic pain after traumatic brain injury a systematic review. Clin Corner 300(6):711-719

2. D'Onofrio F, Russo A, Conte F, Casucci G, Tessitore A, Tedeschi G (2014) Post-traumatic headaches: an epidemiological overview. Neurol Sci 35(SUPPL. 1):203-207

3. Baandrup $L$, Jensen R (2005) Chronic post-traumatic headache - a clinical analysis in relation to the international headache classification 2 nd edition. Cephalalgia. 25(2):132-138

4. Ashina H, Porreca F, Anderson T, Mohammad Amin F, Ashina M, Winther Schytz, et al. Post-traumatic headache: epidemiology and pathophysiological insights. Nat Rev Neurol. 2019; [Epub ahead of print]

5. Seifert TD, Evans RW (2010) Posttraumatic headache: a review. Curr Pain Headache Rep 14(4):292-298

6. Lucas S (2015) Posttraumatic headache: clinical characterization and management. Curr Pain Headache Rep 19(10):1-9

7. Zeeberg P, Olesen J, Jensen R (2005) Efficacy of multidisciplinary treatment in a tertiary referral headache Centre. Cephalalgia. 25(12):1159-1167 
8. Headache Classification Committee of the International Headache Society (IHS) (2018) The international classification of headache disorders, 3rd edition. Cephalalgia. 38(1):1-211

9. Seeger TA, Orr S, Bodell L, Lockyer L, Rajapakse T, Barlow KM. Occipital nerve blocks for pediatric posttraumatic headache: a case series. J child Neurol [internet]. 2015;30(9):1142-1146. Available from: https://doi.org/10. $1177 / 0883073814553973$

10. Dubrovsky AS, Friedman D, Kocilowicz H (2014) Pediatric post-traumatic headaches and peripheral nerve blocks of the scalp: a case series and patient satisfaction survey. Headache. 54(5):878-887

11. Friedman BW, Babbush K, Irizarry E, White D, John Gallagher E. An exploratory study of IV metoclopramide + diphenhydramine for acute posttraumatic headache. Am J Emerg med [internet]. 2018;36(2):285-289. Available from: https://doi.org/10.1016/j.ajem.2017.10.034

12. Chan S, Kurowski B, Byczkowski T, Timm N. Intravenous migraine therapy in children with posttraumatic headache in the ED. Am J Emerg Med [Internet] 2015;33(5):635-639. Available from: https://doi.org/10.1016/j.ajem. 2015.01.053

13. Cushman DM, Borowski L, Hansen C, Hendrick J, Bushman T, Teramoto M (2019) Gabapentin and Tricyclics in the treatment of post-concussive headache, a retrospective cohort study. Headache. 59(3):371-382

14. Kuczynski A, Crawford S, Bodell L, Dewey D, Barlow KM (2013) Characteristics of post-traumatic headaches in children following mild traumatic brain injury and their response to treatment: a prospective cohort. Dev Med Child Neurol 55(7):636-641

15. Erickson JC (2011) Treatment outcomes of chronic post-traumatic headaches after mild head trauma in us soldiers: an observational study. Headache. 51(6):932-944

16. Macedo A, Farré M, Baños JE (2006) A meta-analysis of the placebo response in acute migraine and how this response may be influenced by some of the characteristics of clinical trials. Eur I Clin Pharmacol 62(3):161-172

17. Diener HC, Agosti R, Allais G, Bergmans P, Bussone G, Davies B et al (2007) Cessation versus continuation of 6 -month migraine preventive therapy with topiramate (PROMPT): a randomised, double-blind, placebo-controlled trial. Lancet Neurol 6(12):1054-1062

18. Silberstein SD, Lipton RB, Dodick DW, Freitag FG, Ramadan N, Mathew N et al (2007) Efficacy and safety of topiramate for the treatment of chronic migraine: a randomized, double-blind, placebo-controlled trial. Headache. 47(2):170-180

19. Powers SW, Coffey CS, Chamberlin LA, Ecklund DJ, Klingner EA, Yankey JW et al (2016) Trial of amitriptyline, Topiramate, and placebo for pediatric migraine. N Engl J Med 376(2):115-124

20. Tassorelli C, Diener HC, Dodick DW, Silberstein SD, Lipton RB, Ashina M et a (2018) Guidelines of the international headache society for controlled trials of preventive treatment of chronic migraine in adults. Cephalalgia. 38(5): 815-832

21. Diener HC, Tassorelli C, Dodick DW, Silberstein SD, Lipton RB, Ashina M et al (2019) Guidelines of the international headache society for controlled trials of acute treatment of migraine attacks in adults: fourth edition. Cephalalgia. 39(6):687-710

22. Russo A, D'Onofrio F, Conte F, Petretta V, Tedeschi G, Tessitore A (2014) Post-traumatic headaches: a clinical overview. Neurol Sci 35(SUPPL. 1): 153-157

\section{Publisher's Note}

Springer Nature remains neutral with regard to jurisdictional claims in published maps and institutional affiliations.

Ready to submit your research? Choose BMC and benefit from:

- fast, convenient online submission

- thorough peer review by experienced researchers in your field

- rapid publication on acceptance

- support for research data, including large and complex data types

- gold Open Access which fosters wider collaboration and increased citations

- maximum visibility for your research: over $100 \mathrm{M}$ website views per year

At BMC, research is always in progress.

Learn more biomedcentral.com/submissions 